\title{
Multichannel MAC protocol with dynamic backoff contention for distributed cognitive radio networks
}

\author{
Zong-Heng Wei ${ }^{1,2+}$, Bin-Jie Hu${ }^{2 *}$ and Zhiyong Lin ${ }^{1+}$
}

\begin{abstract}
Multiuser access is a critical issue in distributed cognitive radio networks (DCRNs), as the collisions among the competing secondary users (SUs) may decrease the network throughput significantly. In order to reduce the collisions, we propose a dynamic backoff algorithm to find an optimal backoff contention window to maximize the network throughput and reduce the channel access delay. Based on the proposed algorithm, a multichannel medium access control (MAC) protocol with dynamic backoff contention (MMAC-DB) is designed. In this protocol, the time duration of each channel is divided into four phases: idle, sensing-sharing, contention, and data transmission. The dynamic backoff algorithm is implemented in the contention phase. Moreover, a duration tradeoff is made between the data transmission phase and the contention phase. The effectiveness of the proposed protocol is examined by extensive simulations, which shows that the proposed protocol can enhance the throughput and reduce the channel access delay.
\end{abstract}

Keywords: MAC protocol, Cognitive radio, Dynamic backoff, Access coordination, Throughput, Delay

\section{Introduction}

With the explosive growth of wireless devices and data traffic, the demand for the wireless spectrum resources is greatly increasing. On one hand, the unlicensed spectrum like ISM band has been overcrowded due to the numerous wireless applications; on the other hand, recent spectrum measurements show that significant portion of the licensed spectrum remains unused most of the time [1]. In order to increase the efficiency of spectrum utilization and alleviate the pressure of spectrum demand, cognitive radio (CR) has emerged as a promising technology to realize opportunistic spectrum access (OSA) [2]. In cognitive radio networks (CRNs), unlicensed users (secondary users, SUs) are allowed to opportunistically access the licensed spectrum bands which are temporarily unused by licensed users (primary users, PUs) while not causing harmful interferences to the PUs.

\footnotetext{
*Correspondence: eebjiehu@scut.edu.cn

$\dagger$ Equal Contributor

${ }^{\dagger}$ Zong-Heng Wei, Bin-Jie Hu and Zhiyong Lin contributed equally to this work.

${ }^{2}$ The School of Electronic and Information Engineering, South China University of Technology, Guangzhou, China

Full list of author information is available at the end of the article
}

It is noteworthy that medium access control (MAC) protocol plays a key role in CRNs for OSA [3]. However, the traditional MAC protocols are not suitable for CRNs, because they are based on fixed spectrum allocation, while the available spectrum resource at each SU may vary over frequency/time/space [3]. Therefore, dedicated cognitive radio MAC (CR-MAC) protocols are necessary [4] to realize OSA. Typically, the basic functionalities of a CR-MAC protocol include spectrum sensing, spectrum allocation, spectrum access, spectrum sharing, and spectrum mobility [5].

Recently, distributed CRN (DCRN) has attracted more and more attentions than its centralized counterpart due to its easier and faster deployment and lower cost $[6,7]$. Generally, there are four main objectives to design a distributed CR-MAC [8]: (i) optimization in the spectrum sensing and spectrum access decision, (ii) coordination in the multiuser access in the multichannel network, (iii) optimization in the spectrum resource allocation, and (iv) spectrum trading. However, designing CR-MAC protocols for DCRNs (i.e., distributed CR-MAC protocols) is a very challenging task. This is because DCRNs are not only spectrally heterogeneous but infrastructure-less (i.e., 
without central entity). Without central entity, SUs have to access for spectrum in a randomly competing manner, such as carrier sense multiple access/collision avoidance (CSMA/CA) and ALOHA [9], which inevitably results in collisions among the competing SUs. To reduce the collisions, in this paper, we mainly focus on the multiuser access coordination problem [3] in the design of the distributed CR-MAC protocol. There are two critical issues on multiuser access coordination:

1) How to set the duration of the contention phase?

2) How to coordinate the collided SUs?

For the first issue, we consider a MAC cycle with fixed cycle duration. If the length of the contention phase is relatively short with respect to the number of SUs, the probability of collision will be high, thus decreasing the efficiency of spectrum utilization and increasing channel access delay; on the other hand, if the contention phase costs excessive time, little time is left for data transmission and the ultimate throughput would be unsatisfactory. For the second issue, if each SU is allowed to contend only once in one cycle and the collided SUs (i.e., contention failures) must wait and contend till the next cycle, some potential opportunities of spectrum access would be wasted, and consequently the spectrum access delay would increase. Binary exponential backoff (BEB) algorithm is proposed to decrease the collisions among the SUs and enhance the network throughput in [8]. However, due to the dynamic variations of DCRN, the traditional $\mathrm{BEB}$ algorithm may be not always fit to the various channel contention situations. Therefore, a dynamic backoff scheme is needed to further improve the network throughput and reduce the delay. This is also a new topic in CR-MAC research [5]. In this work, we focus on the design of distributed CR-MAC protocol with efficient dynamic backoff scheme.

Contribution: To optimize the duration of the contention phase and coordinate the collided SUs, we propose a dynamic backoff algorithm in this work. Its main idea is to select the optimal backoff contention window according to the number of contending SUs and the number of idle data channels. Moreover, inspired by the MAC frame structure of the self-scheduled multichannel cognitive radio MAC (SMC-MAC) [10] and the work of [8], we design an efficient CR-MAC protocol with dynamic backoff contention windows for DCRNs. Our major contributions are summarized as follows.

- A dynamic backoff algorithm is proposed to realize the optimal backoff contention windows and to enhance network throughput and decrease channel access delay.

- Based on the dynamic backoff algorithm, a new multichannel CR-MAC protocol called MMAC-DB is proposed for DCRNs. In MMAC-DB, out-of-band control channel (CC) is used and each $\mathrm{SU}$ is equipped with a single radio.

- Extensive simulations are performed to demonstrate the effectiveness of the proposed MMAC-DB protocol in improving the network throughput and reducing channel access delay compared with two existing multichannel MAC protocols in [8] and [10].

The remainder of this paper is organized as follows. Section 2 presents the related work. Section 3 describes the proposed CR-MAC protocol including the details of the dynamic backoff algorithm. Numerical results are given in Section 4. Finally, we conclude our work in Section 5.

\section{Related work}

There are numerous CR-MAC protocols proposed for DCRNs [5, 11-13]. According to [5, 11], these MAC protocols can be broadly classified as single-radio protocols (i.e., split phase) $[8,10,14-17]$ and multiple-radio protocols [18-20]. Using multiple radios can improve the efficiency of channel negotiation process, but it also increases the node complexity as well as deployment cost. Therefore, in this paper, we focus on the cost-efficient single-radio protocols.

A MAC for opportunistic spectrum access (OSA-MAC) is proposed in [14]. In OSA-MAC, the MAC frame structure is composed of a channel selection phase, a sensing phase, and a transmission phase. In the channel selection phase, each SU competes for one channel based on CSMA/CA. However, as only one channel is selected by each SU before sensing, the SUs may select the busy licensed channels instead of idle licensed channels, which decreases the idle channel utilization efficiency. For efficient spectrum utilization, a hardware-constrained MAC (HC-MAC) [15] deals with the channel selection problem with sequential sensing. There are also three phases in HC-MAC: contention phase, sensing phase, and transmission phase. After the CSMA/CA process of the contention phase, a pair of winners will sense multiple licensed channels in a sequential manner and transmit on the sensed idle channels. As there is only one pair of SUs which use multiple channels in HC-MAC, allowing multiple SUs to concurrently utilize the sensed multiple idle channels may further improve the network performance. An opportunistic multi-channel MAC (OMC-MAC) is proposed in [16]. Compared with the aforementioned protocols, OMC-MAC presents a different feature: sensing phase is the first phase, which is followed by the contention phase and the transmission phase. Independent sensing is used in the sensing phase and each SU contends to access the idle licensed channels according to its own sensing results. As independent sensing is inefficient in a multichannel network where each SU must sense the multiple channels, 
sharing the sensing results with other SUs may be more efficient in some cases. In [17], a dedicated common control channel (DCCC)-based distributed multichannel MAC protocol (MMAC-CR) is proposed for multihop CRNs. Each SU is equipped with a dedicated sensing engine which enables sensing concurrently on multiple licensed channels. The sensing results of each SU are contained in a scan result packet (SRP) and shared on the DCCC. Upon receiving the SRP, the SUs contend to access the idle licensed channels in a CSMA/CA manner. However, using dedicated sensing engine adds the cost and the node complexity.

To reduce the cost and the node complexity, a selfscheduled multichannel cognitive radio MAC (SMCMAC) is proposed in [10]. In SMC-MAC, each SU is able to sense only one licensed channel at a time, which makes the radio inexpensive and easy to implement. To reduce the sensing overhead, each SU in SMC-MAC is allowed to sense limited number of licensed channels, then the sensing results are shared with other SUs through the DCCC. Therefore, each SU gets more licensed channel information than that it has sensed. The sensing results are shared on the DCCC during the slotted sensing-sharing (SS) phase. The SS phase is composed of multiple SS slots, and each SS slot is associated with a licensed channel. In each MAC cycle, constrained by the limited sensing capability, each SU randomly picks up several SS slots to sense and share. As more than one SU may pick the same SS slot to share the sensing results, a sensing report collision problem [8] may happen. After that, SUs contend to access the sensed idle channels in the fixed contention phase with a slotted ALOHA manner [21]. A contention problem may happen here, as fixed contention phase may be too short for many SUs, consequently leading to a collision problem, or too long for few SUs, thus wasting the opportunities for data transmission. To handle the contention problem of the fixed contention phase, along the line with SMC-MAC, [8] adds a backoff algorithm to the MAC protocols. With the backoff algorithm, contention phase will be prolonged by adding a backoff window if any collision is detected during the initial contention window. Hence, it can increase the number of successful users substantially and enhance the throughput of DCRNs. However, comparing with the traditional fixed backoff algorithm, using the dynamic backoff algorithm may further improve the network performance [5]. Fu et al., Baher and Doré, and $\mathrm{Wu}$ and $\mathrm{Xu}$ [22-24] have proposed dynamic backoff algorithms for traditional wireless network to improve network throughput and to decrease access delay. But they are not suitable for DCRNs, as they do not consider the interference between PUs and SUs. Therefore, it is still an open problem on how to design a dynamic backoff algorithm and how to set the initial length of the contention phase for DCRNs. Besides, considering more parameters other than the collision results (i.e., number of remaining idle channels) may further improve the backoff algorithm (Table 1).

\section{The proposed MMAC-DB protocol}

In this section, we first introduce the random access protocol, system model and some assumptions. Then, we propose our MMAC-DB protocol by describing its overview as well as detailed operations, with particular focus on

Table 1 Notations used in the paper

\begin{tabular}{|c|c|}
\hline Symbol & Explanations \\
\hline N & Number of SUs in the DCRN \\
\hline$N_{\mathrm{st}}$ & Number of successful transmission pairs in a MAC cycle \\
\hline M & Total number of licensed data channels \\
\hline$M_{\mathrm{ic}}$ & Number of idle licensed channels \\
\hline$M_{\text {sic }}$ & Number of remaining sensed idle licensed channels \\
\hline K & Largest number of slots for the contention phase \\
\hline \multirow[t]{2}{*}{$w_{i}$} & $\begin{array}{l}\text { Average number of winning slots (successful SUs) in the } \\
\text { ith backoff }\end{array}$ \\
\hline & contention window \\
\hline W & Number of slots won in the whole contention phase \\
\hline \multirow[t]{2}{*}{ Q } & $\begin{array}{l}\text { Parameter which is broadcasted on the update slot to } \\
\text { indicate the }\end{array}$ \\
\hline & number of contention slots of the next backoff window \\
\hline$Q_{1}$ & Number of contention slots in the first backoff window \\
\hline$Q_{i}$ & $\begin{array}{l}\text { Number of contention slots in the ith }(i>1) \text { backoff } \\
\text { window }\end{array}$ \\
\hline$Q_{c}$ & $\begin{array}{l}\text { Number of contention slots in the whole contention } \\
\text { phase }\end{array}$ \\
\hline$P_{\mathrm{d}}$ & Each SU's local detection probability \\
\hline$P_{f}$ & Each SU's local false alarm probability \\
\hline$P_{\mathrm{s}}$ & $\begin{array}{l}\text { Probability of a successful RTS contention in the initial } \\
\text { backoff window }\end{array}$ \\
\hline$P_{\mathrm{T}}$ & $\begin{array}{l}\text { Probability of a successful data transmission in a MAC } \\
\text { cycle }\end{array}$ \\
\hline$P_{\mathrm{NT}}$ & Probability of fail to transmit data in a MAC cycle \\
\hline D & Average channel access delay in terms of MAC cycles \\
\hline$T_{\text {cycle }}$ & Time duration of a MAC frame cycle \\
\hline$T_{c}$ & Time duration of contention phase \\
\hline$T_{\mathrm{ct}}$ & $\begin{array}{l}\text { Time duration of contention phase and transmission } \\
\text { phase }\end{array}$ \\
\hline$T_{\mathrm{tr}}$ & Time duration of data transmission phase \\
\hline$\alpha$ & Average utilization probability for all the licensed channels \\
\hline$\sigma$ & Duration of a contention slot in contention phase \\
\hline$R$ & Fixed rate for each data channel \\
\hline$n_{1}$ & $\begin{array}{l}\text { Number of remaining SUs (which still need contention) in } \\
\text { Algorithm } 1\end{array}$ \\
\hline$n_{2}$ & Number of remaining contention slots in Algorithm 1 \\
\hline
\end{tabular}


the dynamic backoff algorithm. Performance analysis is presented at the end.

\subsection{Brief introduction of slotted ALOHA}

The slotted ALOHA protocol is a multiple-node random access protocol, which is a modification of the classical ALOHA protocol [25]. In the slotted ALOHA protocol, all nodes join the competition in an ALOHA period (contention window) which is further divided into multiple contention slots. Each node will randomly choose one contention slot out of the contention window to transmit data. If the slot is occupied by only one node, data would be successfully transmitted; if it is occupied by two or more nodes, there would exist data collisions and data may fail to reach the intended receivers. As the collisions occur within the independent slots only, there are no partial collisions (collisions between two consecutive slots) [21]. Consequently, the collision probability can be significantly reduced compared to the pure ALOHA protocol.

In this paper, we implement cognitive radio ready-tosend (CR-RTS) frame and cognitive radio clear-to-send (CR-CTS) frame [10] with the slotted ALOHA protocol to handle the access coordination problem among the SUs, which is named slotted ALOHA contention scheme. Specifically, to a given contention window with multiple contention slots, an SU (say, SU $i$ ) randomly chooses a contention slot and sends a CR-RTS frame. The CR-RTS frame includes a list of idle channels selected by SU $i$. If SU $i$ is the unique one that has sent out CR-RTS frame in the contention slot, its destination SU (say, $\mathrm{SU} j$ ), upon receiving the CR-RTS frame, will wait for an interframe spacing (CR-SIFS) and then respond a CR-CTS frame which includes the target channel negotiated by $\mathrm{SU} j$. In this way, the pair of source and destination SUs (i.e., SUs $i$ and $j$ ) can successfully complete their channel reservation. Since other SUs can also receive the CR-CTS frame, they are aware of the channel reserved by SUs $i$ and $j$ and will no longer contend for this channel in subsequent contentions. However, if multiple SUs (i.e., SUs $p$ and $q$ ) have chosen the same slot for contention and sent out their CRRTS frames in the slot, a collision may occur and these SUs fail to receive any CR-CTS frame, which means that none of them will win the contention. These collided SUs will retransmit CR-RTS frames in the next contention window. An example of the contention results can be seen in the contention phase in Fig. 1. In Fig. 1, SU $i$ and SU $j$ successfully reserve a data channel during the contention process and transmit data on the reserved channel, while SUs $p$ and $q$ suffer a collision and wait for next contention window to retransmit.

\subsection{System model and assumptions}

We consider a single-hop DCRN which geographically coexists with a primary user (PU) network. The PU network has $M$ orthogonal licensed channels. As in [20], these channels are supposed to have identical propagation characteristics.

The DCRN covers a relatively small area, so that all SUs are assumed to experience the same PU activities. The SUs are allowed to utilize the licensed channels if they are idle (i.e., not used by any PU). Each SU is equipped with a cognitive radio (full-duplex transceiver) which can sense the licensed channels while transmitting data and switching among different channels when necessary. To avoid harmful interferences to the PUs, the SUs need to periodically sense the licensed channels before accessing them. Depending on the hardware capability, we assume each SU can sense only one channel at a time. Using the sequential sensing [26], we can use only one SU to sense each of the licensed channels sequentially and share the sensing results with other SUs, which may avoid the sensing report collision problem. Considering the sensing error effect, similar to [16], we assume each SU has the

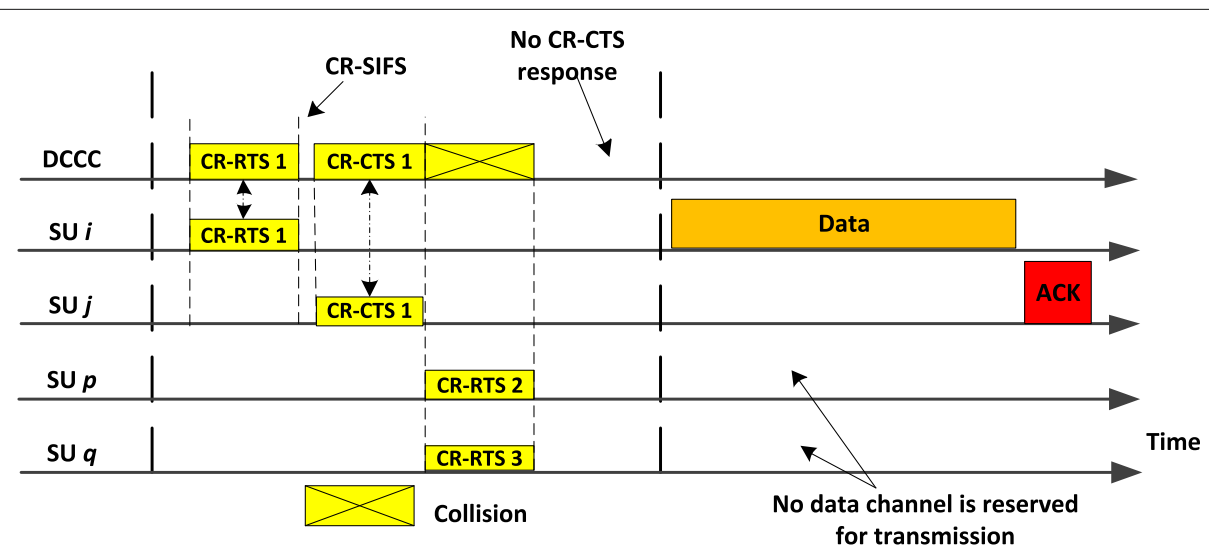

Fig. 1 Slotted ALOHA contention scheme. An example about the slotted ALOHA contention scheme, which is used in the proposed MAC protocol 
same detection probability $\left(P_{\mathrm{d}}\right)$ and false alarm probability $\left(P_{\mathrm{f}}\right)$ on each licensed channel.

We assume that there is an out-of-band channel which can serve as a dedicated common CC (DCCC) for the SUs to exchange control information. Using out-of-band CC is a widely accepted assumption in recent works on distributed CR-MAC protocols [14, 16, 18-20]. The DCCC is assumed to cover all SUs and be always available to them (i.e., without interference from/to PUs). Through the DCCC, the SUs can exchange various control messages. For example, they can easily share their spectrum sensing results. Furthermore, to improve the spectrum utilization efficiency, the DCCC can also be used for data transmission when necessary. That is, at least one idle channel is always available for the SUs to transmit data.

\subsection{Proposed MAC protocol}

\subsubsection{Protocol overview}

The frame structure of our MAC protocol is shown in Fig. 2. The time duration on each channel is divided into cycles (frames), and each cycle $T_{\text {cycle }}$ is further divided into four phases: idle $\left(T_{\text {idle }}\right)$, sensing-sharing $\left(T_{\mathrm{ss}}\right)$, contention $\left(T_{\mathrm{c}}\right)$, and transmission $\left(T_{\mathrm{tr}}\right)$. The sensing-sharing phase is composed of $M$ sensing slots and $M$ sharing slots. Each sensing slot and each sharing slot is associated with a unique licensed channel and has the same time duration as $\tau \mathrm{s}$. In order to handle the access coordination problem and increase the efficiency of idle channel utilization, the contention phase $T_{c}$ is set dynamically in each cycle by the proposed dynamic backoff algorithm. Therefore, to a given MAC cycle, one or multiple backoff contention windows may be used in $T_{c}$. Moreover, each backoff contention window is composed of multiple contention slots to enable slotted ALOHA contention scheme among the SUs. The details will be discussed later in this section.

Considering that our MAC protocol is designed for distributed environments where no central entities exist, to make the protocol feasible and flexible, as in [27, 28], we separate the SUs into two types including manager $\mathrm{SU}$ (MSU) and normal SU (NSU). In each MAC cycle, there should be a MSU which is appointed among the contending SUs with a totally distributed manner and the other SUs become NSUs $[27,28]$. To minimize the control overhead of appointing MSU, in our protocol, the first contention winner in contention phase would act proactively as the MSU in the next MAC cycle. Note that, as there is no first contention winner in the first MAC cycle, SUs will just contend in a predefined contention window without MSU. If a SU is appointed as the MSU, it must perform some managing operations. For example, in each cycle, the MSU should update the protocol parameters (e.g., the length of backoff contention window) and broadcast them on the DCCC. Thereby, the NSUs may receive and update the parameters for every backoff contention process before they choose the contention slots. Considering the control overhead of MSU, as a reward, DCCC will be allocated to the MSU during the transmission phase so that the MSU is contention-free. The detail about how to use DCCC by the MSU will be shown later in Section 3.3.2.

\subsubsection{Operation details}

We introduce the operation details of the four phases of the proposed MAC protocol as follows:

- Idle phase $\left(T_{\text {idle }}\right)$. This is the first phase which has a fixed duration, and it indicates the beginning of a new cycle. During this phase, each SU should turn to the DCCC and wait for the sensing results which will be broadcasted in the subsequent sensing-sharing phase.

- Sensing-sharing phase $\left(T_{\mathrm{ss}}\right)$. During this phase, one of the SUs will sense the licensed channels in a sequential manner. After the sensing process, the sensing SU will switch to the DCCC and broadcast the sensing results one by one. This is a simple way to realize multiple channel sensing without sensing report collision problem. The problem about how to select the sensing $\mathrm{SU}$ for each cycle can be solved by a round robin way, where each SU take turns to sense the licensed channels. Letting the MSU to do the sensing work is another candidate method. Here, we use the MSU to sense the licensed channels in each

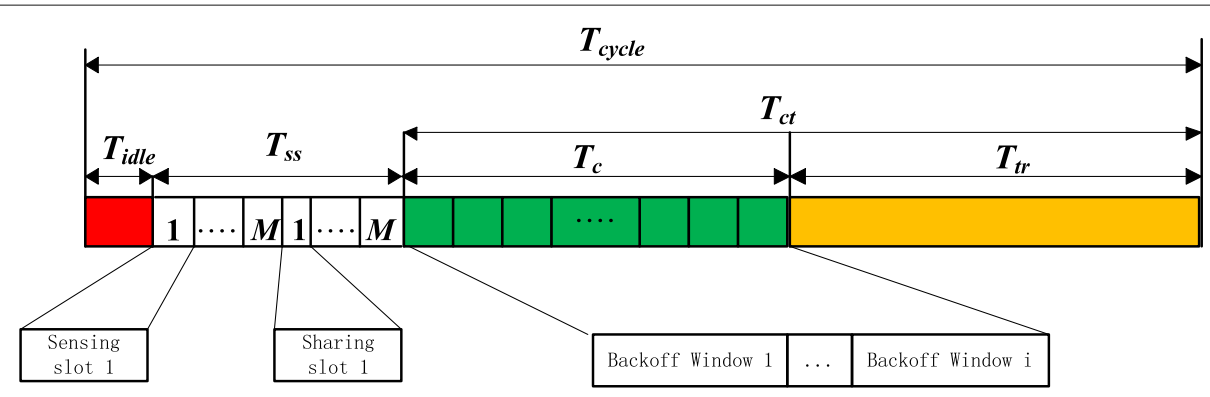

Fig. 2 Frame structure of the proposed MAC protocol. To show the frame structure of the proposed MAC protocol 
cycle. As a new MSU is appointed in every next cycle, the sensing overhead can be shared by the SUs. Moreover, compared with the data transmission phase, the sensing-sharing phase is much shorter, say, to sense 10 channels and to share the sensing results need only $400 \mu s$, which would consume much less time than sending two CR-RTS frames (which takes $600 \mu s)$. Besides, in the contention phase, as MSU only needs to send backoff continue flags and the length of the backoff contention window, the unit time for sending a update frame can be approximated as that for sending a CR-RTS frame. Therefore, being a MSU in one cycle will not generate more power consumption than sending three CR-RTS/CR-CTS frames, which proves the feasibility of the proposed algorithm.

- Contention phase $\left(T_{\mathrm{C}}\right)$. After obtaining the sensing results through the sensing-sharing phase, the SUs will switch to the contention phase and compete to reserve idle channels. As mentioned in the protocol overview, we consider the contention phase as one or multiple backoff contention windows according to the dynamic backoff algorithm (Section 3.3.3). Each backoff contention window consists of an update slot and multiple contention slots. The update slot is specifically used by the MSU to broadcast on the DCCC. The backoff using flag (continue flag) will be broadcasted on the beginning of the update slot. If a continue flag is broadcasted, an additional parameter $Q$ which determines the length of the backoff contention window will be sent by the MSU in the rest time of the update slot. The details about how to set the length of the backoff contention window $Q$ will be discussed in Section 3.3.3. Upon receiving information of the length of the backoff contention window, the SUs will conduct their contentions via the slotted ALOHA contention scheme, which is mentioned before in Section 3.1. During the contention process, MSU will keep listening to the DCCC and execute managing operations. After the contention process, the collided SUs (i.e., contention failures) may continue their contentions in the subsequent backoff windows and wait for the next update slot. The contention phase will be stopped if the MSU stops broadcasting a continue flag on the DCCC. Without receiving the continue flag, all the SUs will stop the contention and move to the transmission phase.

- Transmission phase $\left(T_{\text {tr }}\right)$. The transmission phase starts after the contention phase. In this phase, the SUs which have won the contentions and reserved the idle channels can transmit data on the selected channels. Notice that, to improve the spectrum utilization efficiency, in our protocol the SU which is the first contention winner will use the DCCC to transmit data since the DCCC is always available.

As we focus on the access coordination problem in this paper, the flow chart of the contention phase and transmission phase is shown in Fig. 3. An example about the full operation of the proposed MAC protocol is given in Fig. 4. In Fig. 4, RTS and CTS are short for CR-RTS and CR-CTS. There are five contending SUs in the DCRN: SU1, SU2, SU3, SU4, and SU5. In this MAC cycle, SU5 is the MSU while other SUs are NSUs. At first, all the 5 SUs turn to DCCC during the idle phase. Then, SU5 switches to sense the licensed channels sequentially from 1 to $M$. After that, SU5 shares the sensing results on the reporting slots one by one, from $\mathrm{CH} 1$ to $\mathrm{CH} M$. When all the sensing results are received, SUs move to the contention phase. During the contention phase, at first, SU5 broadcasts the length of the backoff contention window 1 on the first update slot; then, upon the slotted ALOHA contention scheme, during the first backoff contention process, SU2 and SU3 are collided while both SU1 and SU4 win a contention slot to reserve idle data channels. As SU4 is the first winner during the contention phase, it will become the MSU for the next MAC cycle automatically. After that, according to the contention results and dynamic backoff algorithm, SU5 broadcasts the length of the backoff contention window 2 on the second update slot. During the second backoff contention process, both SU2 and SU3 win a contention slot to reserve idle data channels. As there are no more collided SUs, SU5 broadcasts a stop flag on the third update slot to stop the contention phase. Finally, all the SUs move to the transmission phase. During the transmission phase, SU5 will use DCCC for data transmission as a reward for being MSU. Other SUs will switch to the data channels which are reserved during the contention phase.

Next, we will discuss the contention phase in more details since it is the most crucial. Particularly, we will put the focus on how to optimize the backoff values (i.e., number of contention slots within a backoff contention window) of the backoff contention windows.

\subsubsection{Dynamic backoff algorithm}

The dynamic backoff algorithm includes two issues: selecting the initial backoff contention window and selecting the subsequent backoff contention windows.

Initial backoff contention The initial backoff window in each cycle of our protocol consists of $Q_{1}$ contention slots and an update slot. How to determine the value of $Q_{1}$ is important and challenging. A simple solution is to set $Q_{1}$ with a predefined value. However, we may not achieve a 


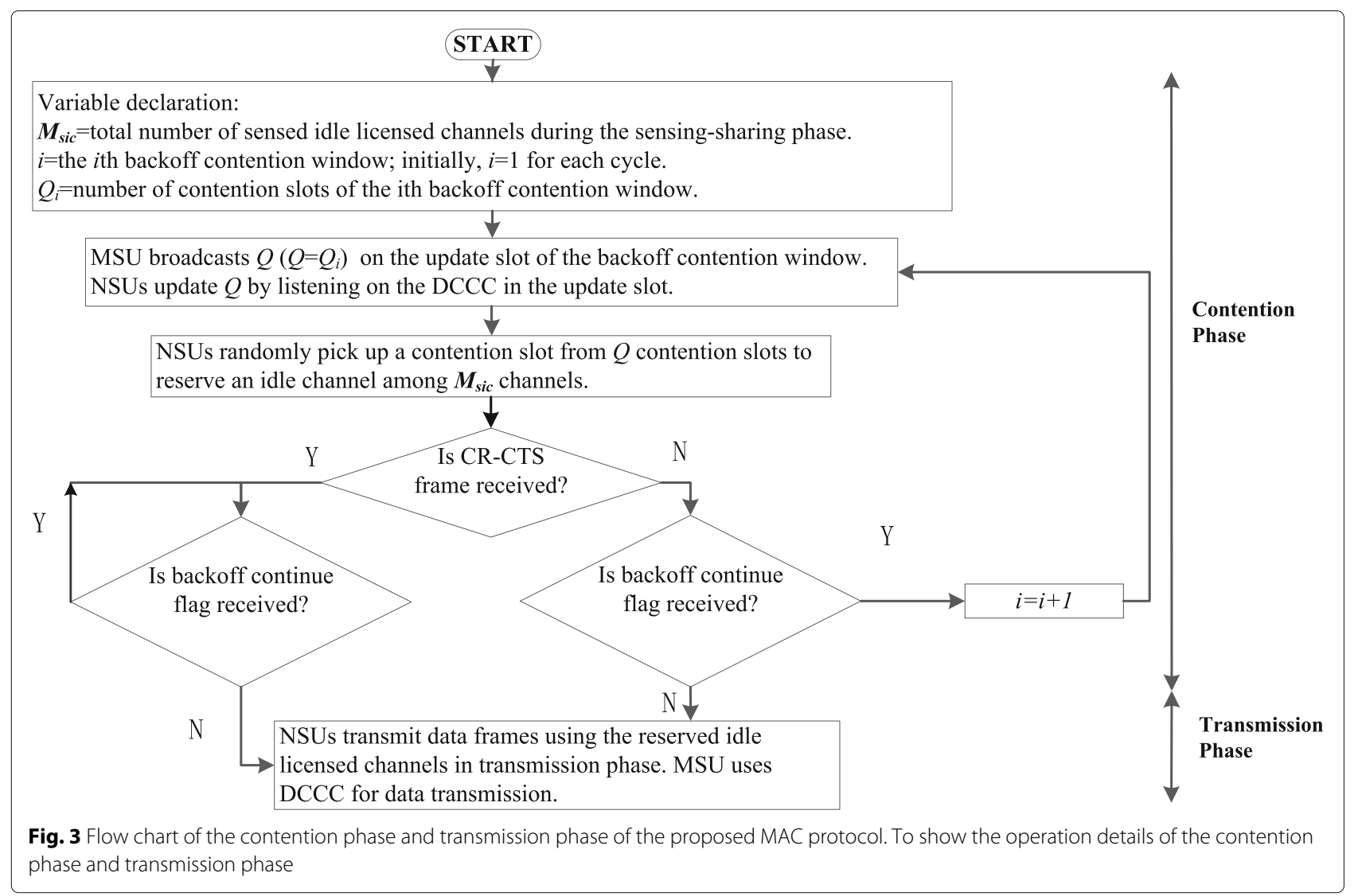

satisfactory system performance by using a fixed $Q_{1}$, especially when the network system contains various numbers of contending SUs and idle channels. Actually, due to the nature of the adopted slotted ALOHA contention scheme, if $Q_{1}$ is too small, few SUs would become contention winners due to the inevitable contention collisions, which may in turn decrease the efficiency of spectrum utilization and increase channel access delay; on the other hand, if $Q_{1}$ is too large, most contention slots may not be used efficiently and less time would be left for data transmission,

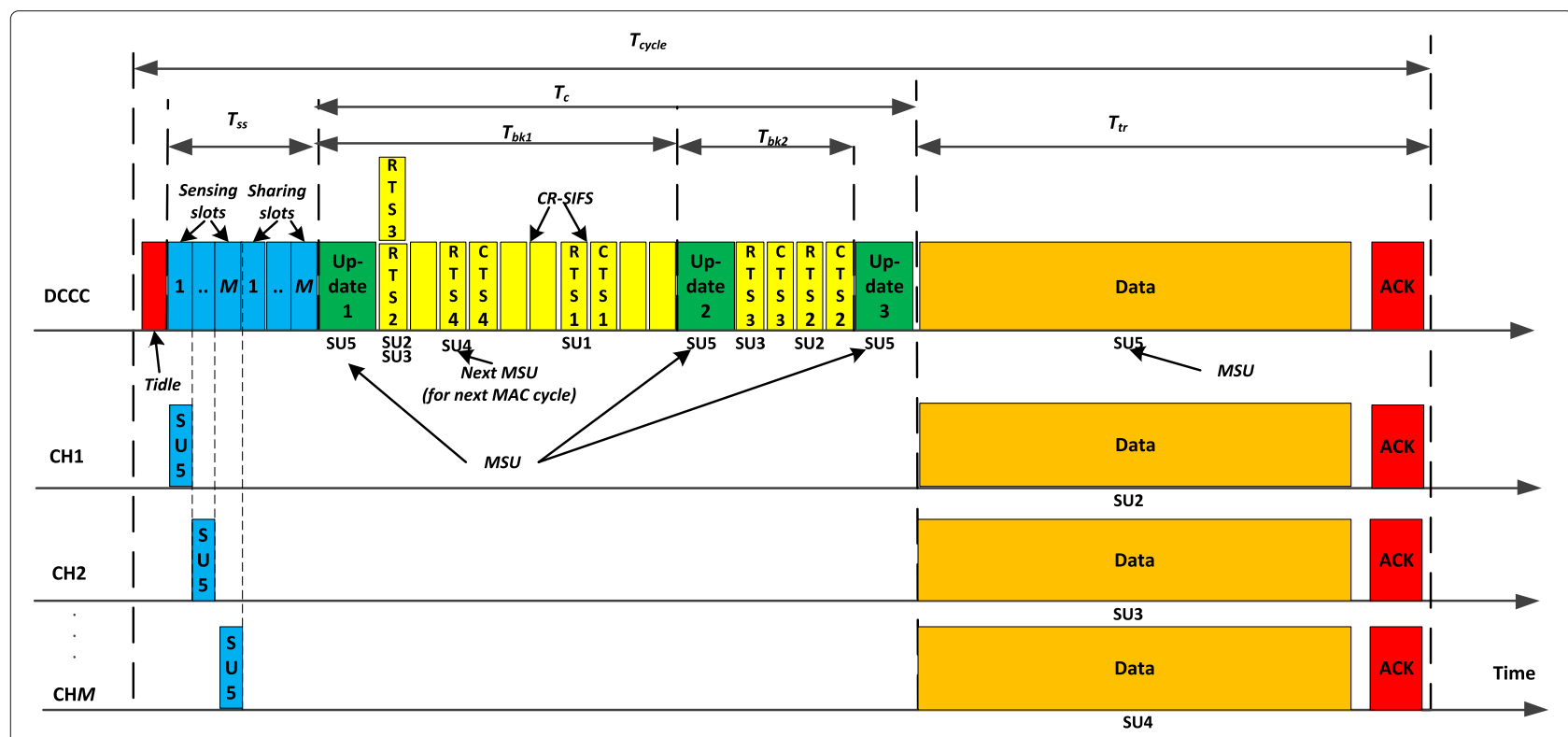

Fig. 4 The operation of the proposed MAC protocol. An example about how the proposed MAC protocol works 
which may also decrease the ultimate system throughput. In view of this, we propose to determine the value of $Q_{1}$ dynamically based on the number of contending NSUs $N$ and the number of remaining sensed idle channels $M_{\text {sic }}$. The basic idea is to select a proper $Q_{1}$ per cycle such that the expected throughput of network can be maximized. The details are as follows.

Given $N, M_{\mathrm{sic}}$, and $Q_{1}$ in a cycle, we first analyze the expected throughput which can be achieved in the initial backoff contention window. As both $N$ and $Q_{1}$ are limited, the initial contention process is a specific slotted ALOHA process, i.e., a frame slotted ALOHA (FSA) process [29]. According to [30-33], FSA process can be viewed as Bernoulli experiments where each SU will select one among the $Q_{1}$ contention slots for contention with a probability $1 / Q_{1}$. Then, the probability for $r$ SUs choosing the same contention slot, denoted by $B(r)$, is derived as follows:

$$
B(r)=\left(\begin{array}{c}
N \\
r
\end{array}\right)\left(\frac{1}{Q_{1}}\right)^{r}\left(1-\frac{1}{Q_{1}}\right)^{N-r}
$$

Given a contention slot, there are only three possible contention results: empty $(r=0)$, successful $(r=1)$, and collision $(r \geq 2)$. Based on Eq. (1), we define $P_{\mathrm{s}}$ as the probability of a contention slot being successful, we have:

$$
P_{\mathrm{s}}=B(1)=\frac{N}{Q_{1}}\left(1-\frac{1}{Q_{1}}\right)^{N-1}
$$

Then, according to [20], we can obtain the average number of successful contention slots $w_{1}$ in the initial backoff contention window:

$$
w_{1}=Q_{1} \times P_{\mathrm{s}}=N\left(1-\frac{1}{Q_{1}}\right)^{N-1}
$$

Considering that $w_{1}$ is also the average number of SUs who have won the contention, the expected throughput $T h_{1}$ for the initial backoff contention can be computed as follows:

$$
T h_{1}=\frac{\left(1+\min \left(w_{1}, M_{\text {sic }}\right)\right)\left(T_{\mathrm{ct}}-\left(Q_{1}+1\right) \sigma\right) R}{T_{\text {cycle }}}
$$

where $R$ is the data rate of each idle channel and $\sigma$ is the time duration of a contention slot. Here, we remark that since the DCCC can also be used for data transmission, we actually have $M_{\mathrm{sic}}+1$ idle channels. Furthermore, $T_{\mathrm{ct}}$ is the duration of both the contention phase and transmission phase (see Fig. 2) and $T_{\mathrm{ct}}=T_{\mathrm{c}}+T_{\mathrm{tr}}$.

Based on Eq. (4), we propose to find the optimal $Q_{1}$ by solving the following discrete optimization problem:

$$
\max _{1 \leq Q_{1} \leq K-1} T h_{1}\left(Q_{1}\right)
$$

where $K=\left\lfloor\frac{T_{\mathrm{ct}}}{\sigma}\right\rfloor$ is the maximal number of slots that can be used for the initial backoff contention window. Let
$Q_{1}^{*}$ denote the optimal $Q_{1}$ of Eq. (4). Here, we give several comments as follows: (i) Given $M_{\text {sic }}$ and $N, Q_{1}^{*}$ can be easily obtained by checking $Q_{1}$ one by one in $O(K)$ time complexity; (ii) We may evaluate the value of $M_{\text {sic }}$ in a cycle based on the sensing results. The value of $N$, if not given in advance, can also be estimated based on the historic contention results by using a method like that of [32]. For the first cycle, if we are unable to know $M_{\text {sic }}$ and $N$, we may set $Q_{1}$ with a predefined value.

Subsequent backoff contentions After the initial backoff contention process, the condition of the network is changed. To use a subsequent backoff contention window, some basic requirements must be met: (i) there are some remaining collided SUs, (ii) there are some remaining sensed idle channels, and (iii) there are some remaining contention slots. If the basic requirements are met, then we need to evaluate the impact on network throughput for using a subsequent backoff window. As adding a subsequent backoff window may bring more successful SUs and less time is left for transmission, we denote the expected throughput with and without the subsequent backoff window as $T h_{\mathrm{WSB}}$ and $T h_{\mathrm{WOSB}}$, respectively. The impact of subsequent backoff window is evaluated by the throughput gain: $\gamma=T h_{\mathrm{WSB}}-T h_{\mathrm{WOSB}}$. As more than one subsequent backoff window may be used for a MAC cycle, given a backoff window $i(i>1)$, we define the length of the current backoff window as $Q_{i}$. Based on the contention analysis of Eqs. (1)-(4), the expected throughput gain $\gamma$ of the backoff window $i$ is:

$\gamma\left(Q_{i}\right)=\frac{\sigma R\left\{\left(\min \left[n_{1}\left(1-\frac{1}{Q_{i}}\right)^{n_{1}-1}, M_{\text {sic }}\right]\left(n_{2}-Q_{i}\right)\right)-(1+W)\left(Q_{i}+1\right)\right\}}{T_{\text {cycle }}}$

where $n_{1}, n_{2}, M_{\text {sic }}$, and $W$ denote the number of SUs which still need contention, the number of remaining slots of the MAC cycle which equals $K-\sum_{i} Q_{i}$, the number of remaining idle channels, and the number of SUs which have won their contention, respectively. The parameters $i, n_{1}, n_{2}, M_{\text {sic }}$, and $W$ can be drawn by the MSU which keeps listening to the DCCC. To make further explanation, Eq. (6) evaluates the throughput gain brought by the $i$ th backoff window (using $Q_{i}$ timeslots for contention). Suppose the $i$ th backoff window is implemented. On one hand, more users may become contention winners and thus have rights to transmit data in the remaining timeslots after the $i$ th backoff window. This will bring a throughput increase, as represented by the term $\sigma R\left(\min \left[n_{1}\left(1-\frac{1}{Q_{i}}\right)^{n_{1}-1}, M_{\text {sic }}\right]\left(n_{2}-Q_{i}\right)\right) / T_{\text {cycle }}$ in Eq. (6). On the other hand, since the existing contention winners (before the $i$ th backoff window) need to refrain from data transmission during the $i$ th backoff window, these users sacrifice their throughput. That is, this 
will bring a throughput decrease, as represented by the term $\sigma R(1+W)\left(Q_{i}+1\right) / T_{\text {cycle }}$ in Eq. (6). The throughput increase minus the throughput decrease yields the throughput gain.

To find the optimal subsequent backoff value, we formulate the following optimization problem:

$$
\max _{1 \leq Q_{i} \leq n_{2}} \gamma\left(Q_{i}\right)
$$

Similar to Eq. (5), the backoff value which can bring the maximum expected throughput gain can be found through an exclusive search process, and the subsequent backoff will continue if the maximum expected throughput gain is larger than 0 .

The detail of dynamic backoff algorithm is given in Algorithm 1.

\subsubsection{Throughput analysis}

We focus on the saturated system through put (i.e., the throughput under the assumption that SUs always have data to transmit). Here, PU activities are assumed to follow a cellular network behavior [34]. In [34], the arrivals of PU calls follow the Poisson distribution and the interarrival time between two calls follow the exponential distribution. Define $T$ as the interarrival time between two PU calls and $\lambda$ as the exponential distribution rate. Then, as the length of each MAC cycle is $T_{\text {cycle, }}$ the maximum interference time between SUs and PUs is $T_{\text {cycle. }}$. The average probability of SUs interfering PUs is [34]:

$$
p_{i}=P\left(T \leq T_{\text {cycle }}\right)=1-\exp \left(-\lambda T_{\text {cycle }}\right)
$$

To a given $p_{i}$, we have $T_{\text {cycle }}=-\ln \left(1-p_{i}\right) / \lambda$.

According to the system model in Section 3.2, we assume that each licensed channel is used by PUs with probability $\alpha$. Let $p(l)$ be the probability of $l$ idle data channels in a MAC cycle, according to [10], we have:

$$
p(l)=\left(\begin{array}{c}
M \\
l
\end{array}\right)(1-\alpha)^{l} \alpha^{M-l}
$$

Then, let $M_{\mathrm{ic}}$ be the average number of idle data channels, $M_{\mathrm{ic}}$ can be expressed as [10]:

$$
M_{\mathrm{ic}}=\sum_{l=0}^{M} l p(l)
$$

As mentioned in Section 3.3.2, all the licensed channels are sensed sequentially by one SU during one cycle. Considering the sensing error effect, the average number of idle channels sensed in one cycle is:

$$
M_{\text {sic }}=M_{\text {ic }} P_{\mathrm{d}}+\left(M-M_{\text {ic }}\right) P_{\mathrm{f}}
$$

After executing Algorithm 1 in the contention phase, during the data transmission phase, SUs turn to the idle data channels they have selected during the contention phase and transmit their data. Since the sensing results and contention results are grabbed through the former

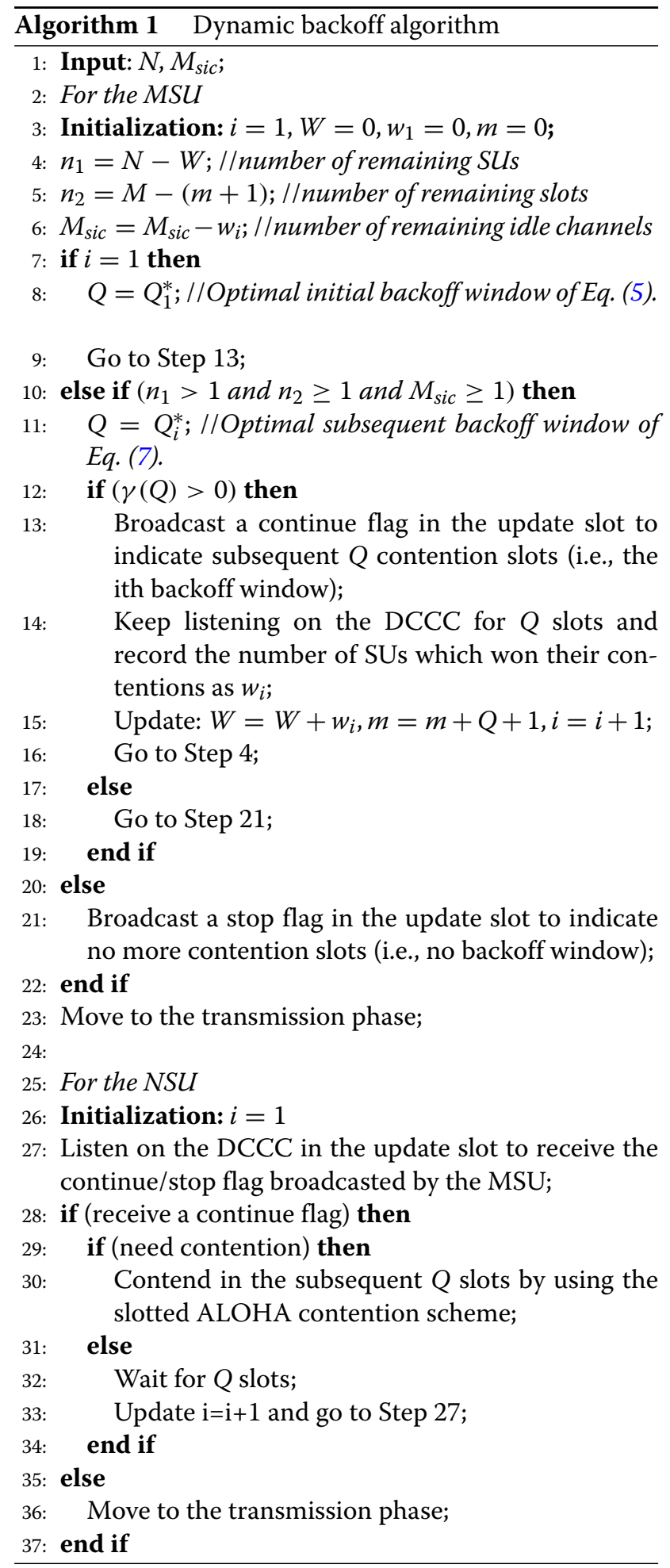

MAC phases, we derive the saturated system throughput by multiplying the number of selected idle channels, the duty cycle for data transmission $\left(T_{\text {tr }} / T_{\text {cycle }}\right)$, and the data rate per sensed idle channel. Considering the missed detection probability, we only consider the successful 
transmissions without interference from PU in throughput calculation as [27]. Then, combining Eq. (11), the saturation throughput of the MMAC-DB protocol is:

$$
T h_{\text {MMAC-DC }}=\frac{\left(1+\min \left(W, M_{\text {sic }}\right) \frac{M_{\text {ic }} P_{\mathrm{d}}}{M_{\text {sic }}}\right) T_{\text {tr }} R}{T_{\text {cycle }}}
$$

where $R$ is the PU interference-free data rate, $W$ is the number of successful SUs, and $T_{\text {tr }}$ is the time duration of data transmission phase which includes the time for acknowledgement packet transmission $[8,10]$. According to Algorithm $1, T_{\text {tr }}$ equals to $n_{2} \sigma$.

\subsubsection{Common control channel bottleneck problem analysis} If too many SUs join a fixed contention phase, the proposed MAC protocol may suffer from the common control channel bottleneck problem (bottleneck problem) [35]: DCCC is overcrowded with too many random access collisions and cannot negotiate for all idle licensed channels. There are mainly two metrics to evaluate the bottleneck problem: collision probability and channel access delay [35]. We will analyze these two metrics as follows.

Collision probability analysis We define the SUs which do not win any contention slot in a MAC cycle as collided SUs. Denoting the average number of collided SUs in a MAC cycle as $N_{c}$, the collision probability can be given as:

$$
P_{\mathrm{c}}=\frac{N_{\mathrm{c}}}{N}=\frac{N-W}{N}
$$

Figure 5 shows the average collision probability of the proposed MAC protocol and two benchmark protocols $[8,10]$. The details about the benchmark protocols will be given in Section 4. We can see the collision probability increases with more contending SUs for all three MAC protocols, which reflects the bottleneck problem. As a
MAC cycle is limited in CR-MAC protocols, the bottleneck problem cannot be avoided. However, the problem can be alleviated by a careful access mechanism design, i.e., using the dynamic backoff mechanism. As can be seen in Fig. 5, the proposed MAC protocol gets a much lower collision probability than the benchmark protocols when $N>80$ and a similar collision probability as [8] when $N<80$.

Channel access delay analysis We analyze the channel access delay of the proposed MAC protocol according to [16]. Similar to [16], we model the data packet transmitting flow as a state diagram in Fig. 6, where each state represents the number of MAC cycles that a packet has been waiting in queue. As we are considering a saturation system, the packet arrival probability of each cycle is 1 , which means the transmitting probability of the initial state of a packet $P_{0}=1$. As channel access delay is an important MAC metric, the sensing error effect is considered here. We assume each SU gets the PU interference-free opportunity to transmit data with a probability $P_{\mathrm{t}}$. Then, we have:

$$
P_{\mathrm{t}}=\frac{1+\min \left(W, M_{\mathrm{sic}}\right) \frac{M_{\mathrm{ic}} P_{\mathrm{d}}}{M_{\text {sic }}}}{N}
$$

Then, the probability of a SU to wait for the next MAC cycle and retransmit data is:

$$
P_{\mathrm{nt}}=1-P_{\mathrm{t}}
$$

Finally, the average channel access delay $(D)$ of the proposed MMAC-DB protocol which is counted in terms of MAC cycles can be given as:

$$
D=\sum_{k=0}^{+\infty} k P_{0} P_{\mathrm{t}} P_{\mathrm{nt}}^{k}=\frac{P_{0} P_{\mathrm{nt}}}{P_{\mathrm{t}}}
$$

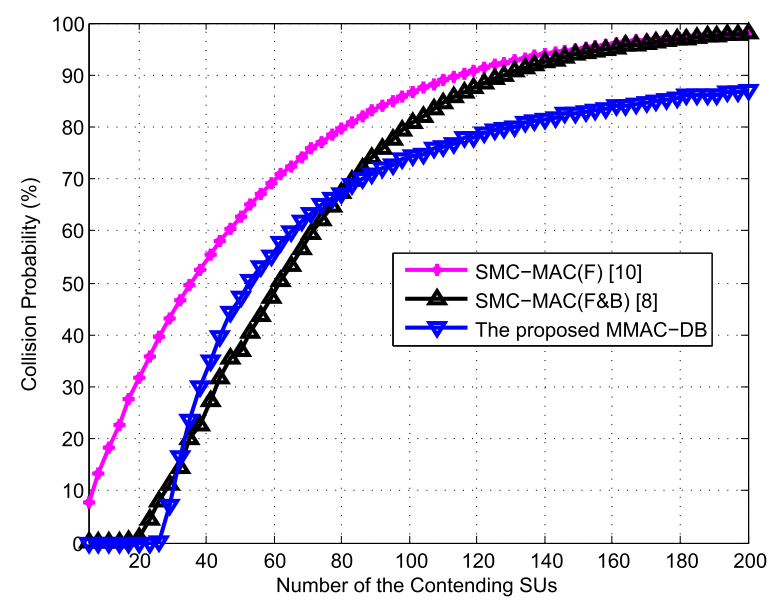

Fig. 5 Collision probability. Collision probability is an important metric to evaluate the control channel bottleneck effect 


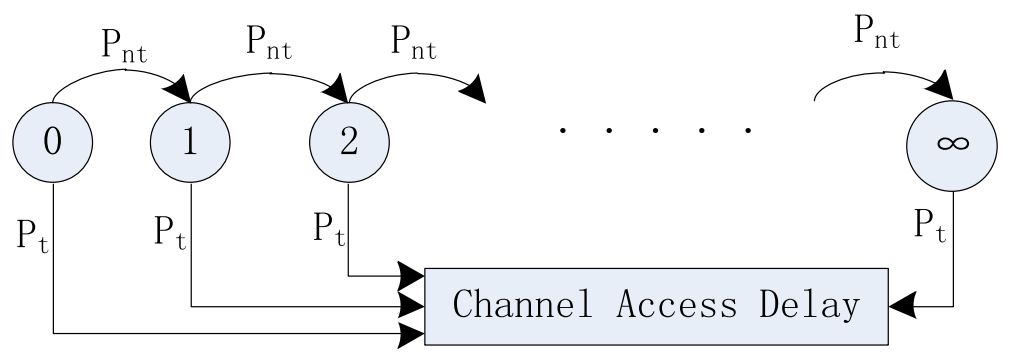

Fig. 6 States of data packets waiting for channel access. State diagram for channel access delay analysis

Channel access delay will be evaluated in three different scenes by extensive simulations in the following Section 4 .

\section{Numerical results}

In this section, numerical results are given to compare the proposed MMAC-DB protocol with two state-of-theart MAC protocols, i.e., SMC-MAC [10] and that of [8]. For convenience and clarity, we hereafter refer to SMC-MAC [10] and that of [8] as SMC-MAC $(F)$ and $\mathrm{SMC}-\mathrm{MAC}(F \& B)$. A summary on these protocols is given in Table 2. Our focus is to verify the efficiency of the proposed protocol by extensive simulations.

We use MATLAB to build the simulator. The simulation settings are as follows. IEEE 802.11 [36] is considered as the physical medium of the MAC protocols. The data rate of each channel $R$ is equal to $1 \mathrm{Mb} / \mathrm{s}$. Sensing-sharing slot time $\tau$ is $20 \mu \mathrm{s}$; CR-RTS, CR-CTS, and CR-SIFS frame times are $300 \mu \mathrm{s}, 300 \mu \mathrm{s}$, and $28 \mu \mathrm{s}$, respectively. $T_{\text {cycle }}=100 \mathrm{~ms}, T_{\text {idle }}=$ CR-SIFS $+2 \times \tau=28 \mu \mathrm{s}, T_{\mathrm{ss}}=2 \times$ $M \times \tau=M \times 40 \mu s, \sigma=T_{\mathrm{CR}-\mathrm{RTS}}+T_{\mathrm{CR}-\mathrm{CTS}}+$ $T_{\mathrm{CR}-\mathrm{SIFS}}=628 \mu \mathrm{s}$. For both SMC-MAC $(F)$ and SMC$\operatorname{MAC}(F \& B)$, their initial backoff windows consist of the same number of slots which is denoted by $K_{f}=50$. Then, we set the number of contention slots of the initial backoff value of binary exponential backoff (BEB) window as $B_{1}=16$ as [8]. Moreover, in SMC-MAC $(F \& B)$, the backoff process will be stopped when all the idle channels are reserved or there is no time left for new backoff window in this MAC cycle. We vary the three key parameters, i.e., PU traffic load $\alpha$, number of licensed data channels $M$ and number of contending SUs $N$, by considering three different scenes. Moreover, to evaluate the sensing error effect, we set $P_{\mathrm{d}}=1, P_{\mathrm{f}}=0$ for perfect

Table 2 Summary on the compared protocols

\begin{tabular}{lll}
\hline Protocol & $\begin{array}{l}\text { Initial backoff } \\
\text { window }\end{array}$ & $\begin{array}{l}\text { Subsequent backoff } \\
\text { windows }\end{array}$ \\
\hline SMC-MAC $(F)[10]$ & Fixed length & No backoff \\
SMC-MAC $(F \& B)[8]$ & Fixed length & $\begin{array}{l}\text { Using binary } \\
\text { exponential backoff }\end{array}$ \\
The proposed MMAC-DB & Dynamic length & $\begin{array}{l}\text { Using dynamic } \\
\text { backoff }\end{array}$ \\
\hline
\end{tabular}

sensing and set $P_{\mathrm{d}}=0.9, P_{\mathrm{f}}=0.1$ for imperfect sensing in each scene. For each set of parameter values, simulation results are obtained with running 10000 MAC cycles. Next, we present the numerical results for each scene.

\subsection{Scene 1: Influence of $\alpha$ for fixed $N$ and $M$}

Figures 7 and 8 show the throughput and channel access delay comparison of different protocols under different PU traffic load $\alpha$. As showed in Fig. 7, the proposed MMAC-DB has the best throughput performance under both perfect and imperfect sensing settings. As expected, considering the interferences with PUs, imperfect sensing decreases the throughput of the proposed MMAC-DB compared to that under perfect sensing. Moreover, in general, to all the MAC protocols, the throughput decreases with higher PU traffic loads, due to less idle data channels. Furthermore, when $\alpha \leq 0.3$, the throughput of SMC$\operatorname{MAC}(F \& B)$ is a bit lower than that of SMC-MAC $(F)$. This is because of the nature of the traditional $B E B$ algorithm: when there are plenty of idle data channels, it will spend too much time for using the backoff windows without considering the throughput gain. Also, we observe from Fig. 7 that all the three protocols achieve good throughput performance when $\alpha$ is low. Then, we focus on the low PU traffic load $(\alpha=0.1)$ case in the following scenes. From Fig. 8, we can see that the channel access delay of the proposed MAC protocol is lower than that of the other two MAC protocols. This is due to that the proposed dynamic backoff algorithm conducts the contention phase in each MAC cycle adaptively and guarantees that the contending SUs have a high successful probability to transmit data.

\subsection{Scene 2: Influence of $M$ for fixed $N$ and $\alpha$}

Figures 9 and 10 demonstrate the performance of different protocols in terms of throughput and channel access delay under various $M$. Generally speaking, from Fig. 9, we can see that the throughputs of all the MAC protocols increase with larger $M$. The proposed MMAC-DB protocol reaches a throughput upper bound when $M \geq 22$ under perfect sensing. Considering the imperfect sensing, 


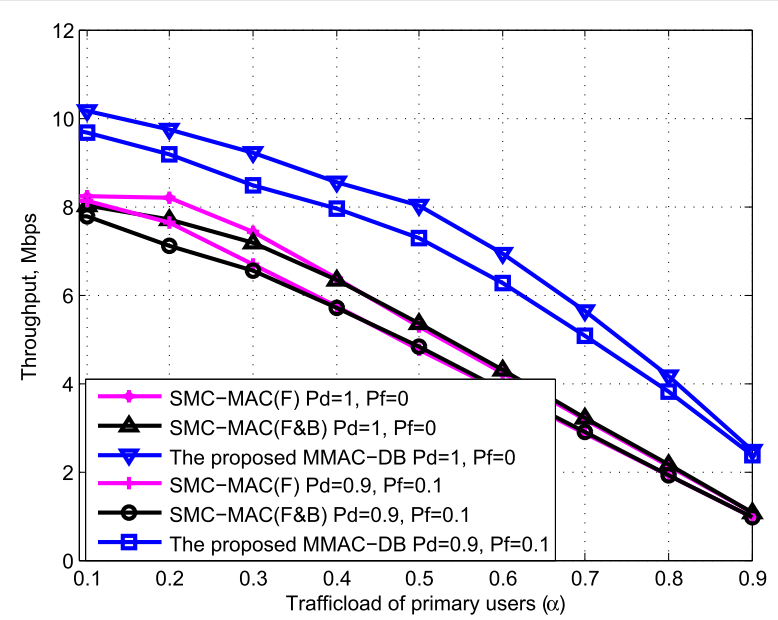

Fig. 7 Throughput comparison with varying PU traffic load, $N=20, M=20, K_{f}=50$. Throughput comparison for scene 1

we can see a throughput upper bound of the proposed MMAC-DB is achieved when $M \geq 25$. This is because that the number of sensed idle channels is larger than the number of contending SUs, and therefore increasing $M$ would bring no more throughput gain. Also, due to the fixed initial contention window and the lack of subsequent backoff windows, SMC-MAC $(F)$ gets a much lower throughput upper bound than that of the proposed MMAC-DB. Moreover, there are some fluctuations of values for SMC-MAC $(F \& B)$ when $M \geq 25$, and the throughput of SMC-MAC $(F \& B)$ is higher under imperfect sensing than that under perfect sensing. This is due to that, in SMC-MAC $(F \& B)$, if there are still some idle channels and collided SUs, the contention process will run on and more backoff windows will be used. As the number of licensed data channel increases, a new backoff window will be used even if there is only one idle channel which is not reserved in the former backoff windows. Therefore, adding a new backoff window would be inefficient in some cases. In Fig. 9, as more idle channels are sensed under perfect sensing, more backoff windows are used and less time is left for transmission, which decreases the throughput of SMC-MAC $(F \& B)$. Figure 9 illustrates that to design an access coordination mechanism properly is very important especially when the spectrum resource is plenty. As expected, in Fig. 10, we can see the channel access delay decreases as $M$ increases for that more idle data channels bring higher channel access probability. The proposed MMAC-DB has the lowest channel access delay. Due to the backoff scheme, the channel access delay of SMC-MAC $(F \& B)$ is much lower than that of SMC-MAC $(F)$.

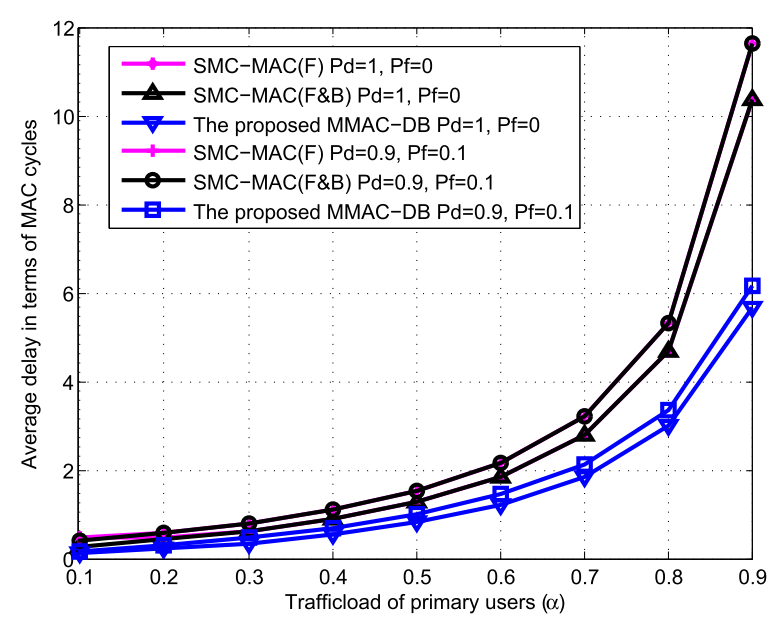

Fig. 8 Channel access delay comparison with varying PU traffic load, $N=20, M=20, K_{f}=50$. Channel access delay comparison for scene 1 


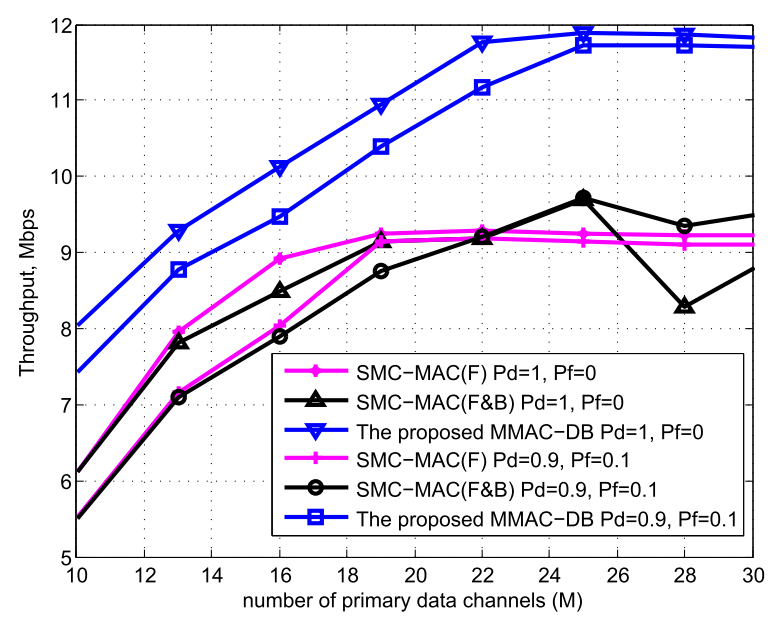

Fig. 9 Throughput comparison with varied $M$ from 10 to $30, N=20, \alpha=0.1$. Throughput comparison for scene 2

\subsection{Scene 3: Influence of $N$ for fixed $M$ and $\alpha$}

As $N$ is the crucial parameter to evaluate the dynamic nature of all the MAC protocols, more details are given here besides the network performance. Figures 11, 12, and 13 show three MAC metrics: the average number of sensed idle channels $\left(M_{\text {sic }}\right)$, the average number of contention slots of the contention phase $\left(Q_{c}\right)$, and the average number of successful transmission pairs $\left(N_{\text {st }}\right)$ in a MAC cycle. In Fig. 11, with the sequential sensing scheme, the $M_{\text {sic }}$ of the proposed MMAC-DB protocol is unaffected by various $N$ and is higher than that of the other two protocols when $N<80$. As expected, in Fig. 12, the $Q_{\mathrm{c}}$ changes with $N$ except for SMC-MAC $(F)$, which shows the influence of various $N$ on the contention phase setting. From Fig. 12, to both of the proposed MMAC-DB protocol and SMC-MAC $(F \& B), Q_{\mathrm{c}}$ increases with higher
$N$ when $N$ is smaller than the number of sensed idle channels. This is because that, in this case, any SU can grab an idle channel if it wins the contention. Therefore, to increase the throughput gain, the collision probability should be kept very low, which can be seen in the former Fig. 5. To achieve a low collision probability, more contention slots are needed here. When $N$ is larger than the number of sensed idle channels, there are not enough idle channels for all the $N$ SUs. Hence, considering the throughput gain, just a part of the $N$ SUs should access the insufficient idle channels, and a higher collision probability is allowed. In this case, fewer contention slots are needed with the increasing $N$ until $N$ is high enough to cause a bottleneck problem. As $N$ keeps increasing, if $Q_{c}$ is fixed, the bottleneck problem will become serious and the collision probability will be high. To alleviate the

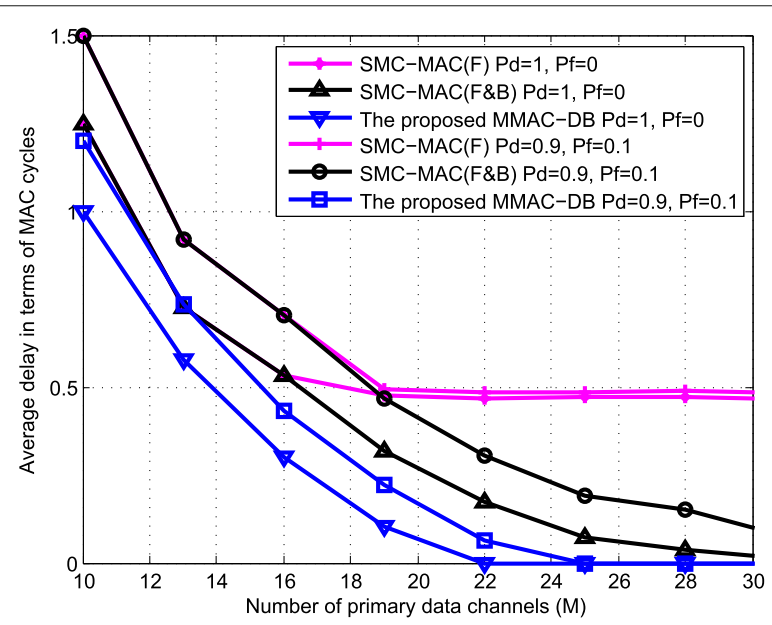

Fig. 10 Channel access delay comparison with varied $M$ from 10 to $30, N=20, \alpha=0.1$. Channel access delay comparison for scene 2 


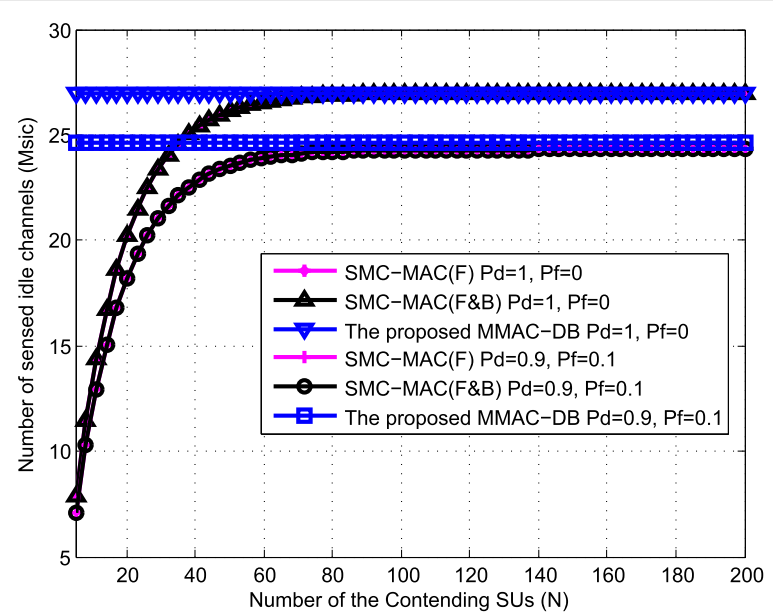

Fig. 11 Number of sensed idle data channels with $M=30, \alpha=0.1$. Sensed idle data channels analysis for scene 3

bottleneck problem, $Q_{\mathrm{c}}$ increases again when $N$ is around 70 , as more contention slots are needed to coordinate the collisions.

The impact of $N_{\text {st }}$ under various $N$ is shown in Fig. 13. When $N$ is small, the number of successful transmission pairs is increasing at a very high rate with the increasing $N$. The $N_{\text {st }}$ of the proposed MMAC-DB remains stable when it is near the number of idle data channels. This is due to that the proposed dynamic backoff mechanism may adaptively adjust the length of backoff window by taking into account network situational factors including the number of remaining idle channels, the number of contending users, and the number of remaining timeslots of a cycle. Particularly, when there are number of users contending relatively sufficient resources (i.e., idle channels and remaining timeslots), the proposed dynamic mechanism tends to allocate a backoff window with large length so as to make more users obtain successful contention. However, we can see a sharp decrease of the SMC-MAC $(F \& B)$ when it is close to the number of idle data channels. This is because SMC-MAC $(F \& B)$ just simply doubles the length of the backoff window when backoff is needed. In such strategy, the remaining time in a cycle may be run out quickly; as a result, no more backoff window can be allocated even when there are still many users that want to continue the contention. In other words, the simple backoff mechanism adopted in SMC-MAC $(F \& B)$ still cannot accommodate large number of contending users well.

Throughput performance is shown in Fig. 14. Under the large dynamic range of $N$, to all the three MAC protocols, MMAC-DB has the optimal throughput performance.

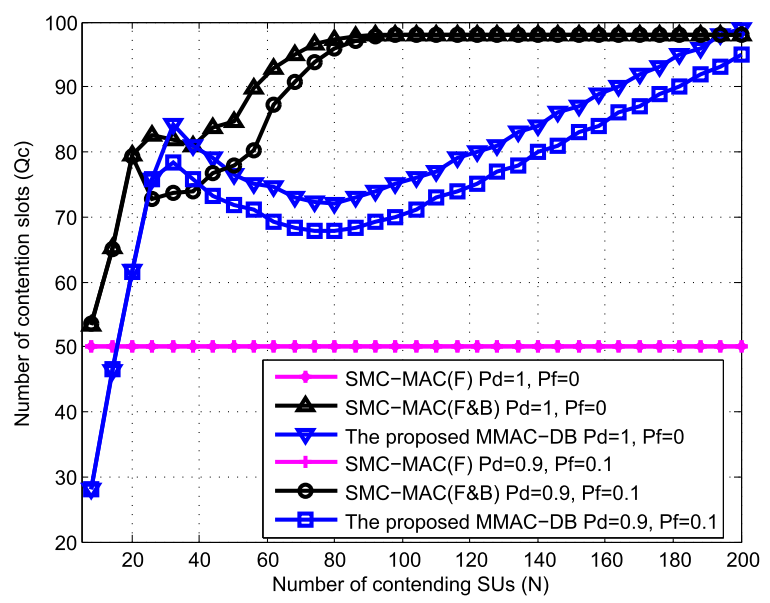

Fig. 12 Number of contention slots with $M=30, \alpha=0.1$. Contention window analysis for scene 3 


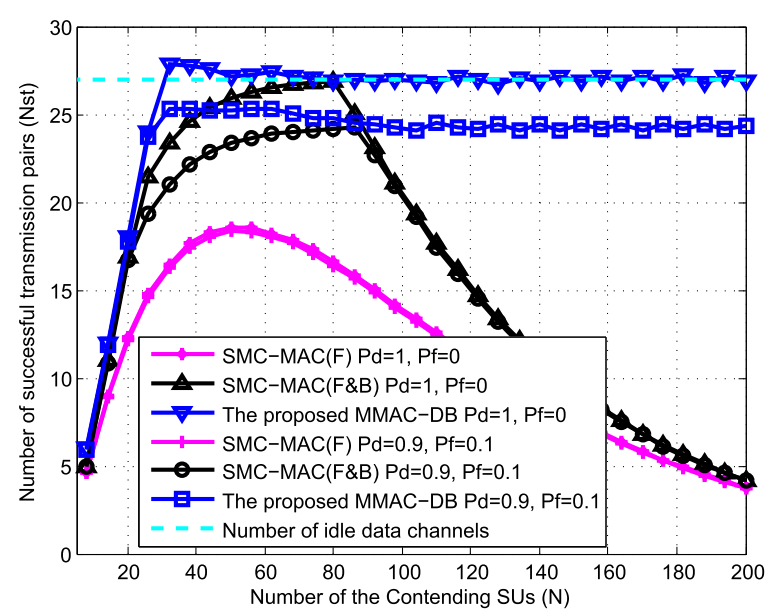

Fig. 13 Number of successful transmission pairs with $M=30, \alpha=0.1$. The number of the successful transmission pairs in scene 3

Moreover, we can see the throughput of MMAC-DB reaches a peak when $N$ is around 70 and gradually decreases when $N>70$. This is due to the bottleneck problem that more SUs cost more contention slots to coordinate the collisions, leaving less time for data transmission. Although the bottleneck problem cannot be avoided, the proposed MMAC-DB protocol alleviates the bottleneck problem and achieves a good throughput performance when $N$ is large.

Channel access delay performance is shown in Fig. 15. From Fig. 15, we can see a sharp increase for SMC$\operatorname{MAC}(F)$ when $N>60$. Also, there is a similar sharp increase for SMC-MAC $(F \& B)$ when $N>100$. The above facts are due to that SMC-MAC $(F)$ and SMC-MAC $(F \& B)$ are incapable to coordinate so many contending SUs and to alleviate the bottleneck problem. The channel access delay of the proposed MMAC-DB is much lower than that of the other two protocols.

\section{Conclusion}

In this paper, we presented a dynamic backoff algorithm to reduce the collisions among the contending SUs. Based on the proposed algorithm, we further developed a multichannel CR-MAC protocol with a dynamic backoff scheme (MMAC-DB) for distributed cognitive radio network. The proposed protocol is flexible enough to fit for a changing DCRN environment with a good throughput performance by dynamically setting the backoff contention windows. As the collisions among the SUs are reduced by the proposed protocol, the channel access delay is also reduced. To sum up, with the proposed MMAC-DB protocol, both the throughput and

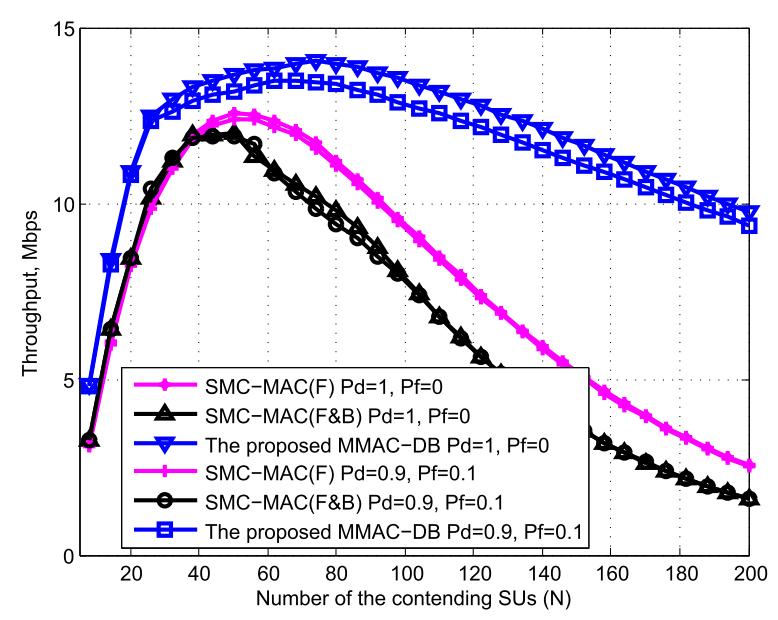

Fig. 14 Throughput comparison with $M=30, \alpha=0.1$. Throughput comparison for scene 3 


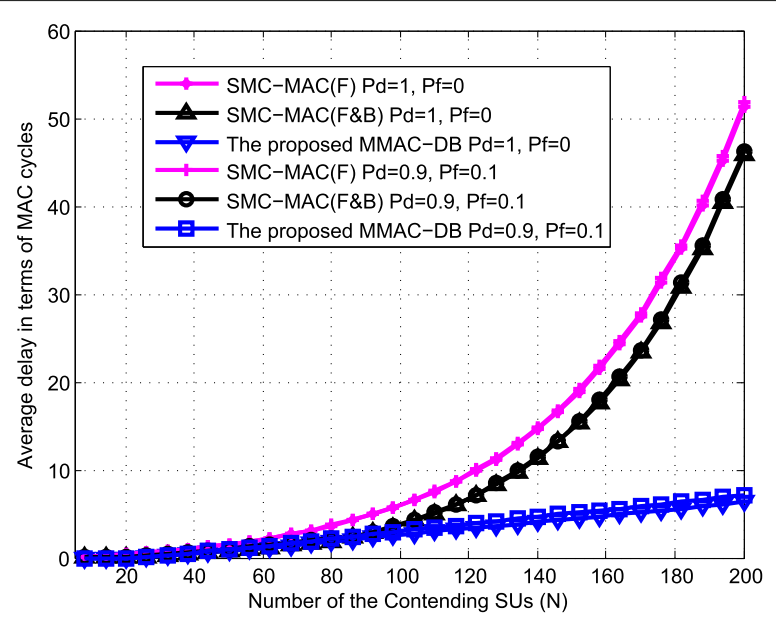

Fig. 15 Channel access delay comparison with $M=30, \alpha=0.1$. Channel access delay comparison for scene 3

the channel access delay of the distributed cognitive radio network are enhanced compared with those of the two existing CR-MAC protocols, which is demonstrated by extensive simulations and the network throughput improvement is up to $282.75 \%$ under perfect sensing scenario and is up to $272.37 \%$ under imperfect sensing scenario.

\section{Authors' contributions}

ZHW is the first author of the manuscript. He designed the study and developed the protocol. In addition, he did the performance evaluations and numerical result studies, and wrote the manuscript. BJH is the second author of the manuscript. He helped to design the study and develop the protocol. He also revised the manuscript. ZYL is the third author of the manuscript. He helped to do the performance evaluations and numerical result studies. He also revised the manuscript. All of the authors take full responsibility for the content of the paper. All authors read and approved the final manuscript.

\section{Funding}

This work was supported by the national natural science foundation of China (NSFC) under Grant 61871193 and 61202453 and by the key project of guangdong natural science foundation under Grant 2018B030311049.

\section{Competing interests}

The authors declare that they have no competing interests.

\section{Author details}

${ }^{1}$ School of Computing Science, Guangdong Polytechnic Normal University, Guangzhou, China. ${ }^{2}$ The School of Electronic and Information Engineering, South China University of Technology, Guangzhou, China.

Received: 16 November 2017 Accepted: 11 July 2019

Published online: 07 August 2019

\section{References}

1. FCC Spectrum Policy Task Force, Report of the spectrum efficiency working group (2002). https://transition.fcc.gov/sptf/files/ SEWGFinalReport 1.pdf

2. Y. C. Liang, K. C. Chen, G. Y. Li, P. Mahonen, Cognitive radio networking and communications: an overview. IEEE Trans. Veh. Technol. 60(7), 3386-3407 (2011)

3. P. Ren, Y. Wang, Q. Du, J. Xu, A survey on dynamic spectrum access protocols for distributed cognitive wireless networks. EURASIP J. Wirel. Commun. Netw. 2012(1), 60 (2012)
4. L. Gavrilovska, D. Denkovski, V. Rakovic, M. Angjelichinoski, Medium access control protocols in cognitive radio networks: overview and general classification. IEEE Commun. Surv. Tutor. 16(4), 2092-2124 (2014)

5. A. Sultana, X. Fernando, L. Zhao, An overview of medium access control strategies for opportunistic spectrum access in cognitive radio networks. Peer-to-Peer Netw. Appl. 10(5), 1113-1141 (2017)

6. C. Cormio, K. R. Chowdhury, A survey on MAC protocols for cognitive radio networks. Ad Hoc Netw. 7(7), 1315-1329 (2009)

7. S. C. Jha, M. M. Rashid, V. K. Bhargava, C. Despins, Medium access control in distributed cognitive radio networks. IEEE Wirel. Commun. 94-A(4), 41-51 (2011)

8. S. Pandit, G. Singh, Backoff algorithm in cognitive radio MAC protocol for throughputenhancement. IEEE Trans. Veh. Technol. 64(5), 1991-2000 (2015)

9. A. C. V. Gummalla, J. O. Limb, Wireless medium access control protocols. IEEE Commun. Surv. Tutor. 3(2), 2-15 (2000)

10. S. Lim, L. Tae-Jin, A self-scheduling multi-channel cognitive radio MAC protocol based on cooperative communications. leice Trans. Commun. 94-B(6), 1657-1668 (2011)

11. A. D. Domenico, E. C. Strinati, M. G. D. Benedetto, A survey on MAC strategies for cognitive radio networks. IEEE Commun. Surv. Tutor. 14(1), 21-44 (2012)

12. M. G. D. Benedetto, F. Bader, Cognitive Communication and Cooperative HetNet Coexistence. (Springer, Switzerland, 2014)

13. M. G. D. Benedetto, A. F. Cattoni, J. Fiorina, F. Bader, L. D. Nardis, Cognitive Radio and Networking for Heterogeneous Wireless Networks. (Springer, Switzerland, 2015)

14. L. Le, E. Hossain, in Wireless Communications and NETWORKING Conference, 2008. A mac protocol for opportunistic spectrum access in cognitive radio networks (WCNC, 2008), pp. 1426-1430

15. J. Jia, Q. Zhang, X. Shen, HC-MAC: a hardware-constrained cognitive mac for efficient spectrum management. Sel. Areas Commun. IEEE J. 26(1), 106-117 (2008)

16. S. C. Jha, U. Phuyal, M. M. Rashid, V. K. Bhargava, Design of OMC-MAC: an opportunistic multi-channel MAC with QoS provisioning for distributed cognitive radio networks. IEEE Trans. Wirel. Commun. 10(10), 3414-3425 (2011)

17. M. Timmers, e. Pollin, A distributed multichannel MAC protocol for multihop cognitive radio networks. IEEE Trans. Veh. Technol. 59(1), 446-459 (2010)

18. H. Su, X. Zhang, Cross-layer based opportunistic MAC protocols for QoS provisionings over cognitive radio wireless networks. Sel. Areas Commun. IEEE J. 26(1), 118-129 (2008)

19. W. S. Jeon, J. A. Han, G. J. Dong, A novel MAC scheme for multichannel cognitive radio ad hoc networks. IEEE Trans. Mob. Comput. 11(6), 922-934 (2012)

20. S. Debroy, S. De, M. Chatterjee, Contention based multichannel MAC protocol for distributed cognitive radio networks. Mob Comput. IEEE Trans. 13(12), 2749-2762 (2014) 
21. D. K. Klair, K. W. Chin, R. Raad, A survey and tutorial of RFID anti-collision protocols. IEEE Commun. Surv. Tutor. 12(3), 400-421 (2010)

22. J. Fu, Q. Zhang, H. Wang, in 20095 th International Conference on Wireless Communications, Networking and Mobile Computing, IEEE, Beijing. A new backoff algorithm based on the dynamic modulating parameters of ieee 802.11, (2009), pp. 1-4

23. M. Baher, J.-B. Doré, in Multiple Access Communications. CSMA/CA bottleneck remediation in saturation mode with new backoff strategy (Springer, Cham, 2013), pp. 70-81

24. G. Wu, P. Xu, Improving performance by a dynamic adaptive success-collision backoff algorithm for contention-based vehicular network. IEEE Access. 6, 2496-2505 (2018)

25. N. Abramson, in November 17-19, 1970, Fall Joint Computer Conference. The aloha system:another alternative for computer communications, (1970), pp. 281-285

26. D. T. C. Wong, S. Zheng, Y. C. Liang, in IEEE International Conference on Communications, IEEE, Kyoto, 2011. Cognitive multi-channel mac protocols with perfect and imperfect sensing, (2011), pp. 1-5

27. B. Hamdaoui, K. G. Shin, OS-MAC: an efficient mac protocol for spectrum-agile wireless networks. IEEE Trans. Mob. Comput. 7(8), 915-930 (2008)

28. S. Kwon, B. Kim, B. h.Roh, Preemptive opportunistic mac protocol in distributed cognitive radio networks. IEEE Commun. Lett. 18(7), 1155-1158 (2014)

29. H. Okada, Y. Igarashi, Y. Nakanishi, Analysis and application of framed aloha channel in satellite packet switching networks - fadra method. Electron. Commun. Jpn. 60, 72-80 (1977)

30. K. Elleithy, T. Sobh, New Trends in Networking, Computing, E-learning, Systems Sciences, and Engineering. (Springer, Switzerland, 2015)

31. S. R. Lee, S. D. Joo, C. W. Lee, in International Conference on Mobile and Ubiquitous Systems: NETWORKING and Services, IEEE Computer Society, San Diego, 2005. An enhanced dynamic framed slotted aloha algorithm for rfid tag identification (MOBIQUITOUS, 2005), pp. 166-172

32. W. T. Chen, An accurate tag estimate method for improving the performance of an RFID anticollision algorithm based on dynamic frame length ALOHA. IEEE Trans. Autom. Sci. Eng. 6(1), 9-15 (2009)

33. J. B. Eom, T. J. Lee, Accurate tag estimation for dynamic framed-slotted ALOHA in RFID systems. IEEE Commun. Lett. 14(1), 60-62 (2010)

34. D. Willkomm, S. Machiraju, J. Bolot, A. Wolisz, Primary user behavior in cellular networks and implications for dynamic spectrum access. IEEE Commun. Mag. 47(3), 88-95 (2009)

35. G. P. Joshi, S. Y. Nam, S. W. Kim, An analysis of channel access delay in synchronized mac protocol for cognitive radio networks. Trans. Emerg. Telecommun. Technol. 25(5), 485-489 (2014)

36. IEEE Standard for Information Technology - Telecommunications and Information Exchange Between Systems - Local and Metropolitan Area Networks - Specific Requirements - Part 11, Wireless LAN Medium Access Control (MAC) and Physical Layer (PHY) Specifications. IEEE Std 802.11-2007 (Revision of IEEE Std 802.11-1999), 1-1076 (2007). IEEE Std. 802.11

\section{Publisher's Note}

Springer Nature remains neutral with regard to jurisdictional claims in published maps and institutional affiliations.

\section{Submit your manuscript to a SpringerOpen ${ }^{\odot}$ journal and benefit from:}

- Convenient online submission

- Rigorous peer review

- Open access: articles freely available online

- High visibility within the field

- Retaining the copyright to your article

Submit your next manuscript at $\gg$ springeropen.com 